\title{
Physician Perception of Patient Medication Adherence in a Cohort of Medicare Advantage Plans in Texas
}

\author{
Amanda Winters, PharmD; Tara Esse, PharmD, BCACP; Archita Bhansali, MS; \\ Omar Serna, PharmD, BCACP; Shivani Mhatre, PhD; and Sujit Sansgiry, PhD
}

\begin{abstract}
BACKGROUND: Prescription medication adherence is a known health-related barrier for elderly patients, leading to insufficient disease control and negative health outcomes. The Centers for Medicare \& Medicaid Services (CMS) have placed significant emphasis on medication adherence, through the Part $D$ star measures, revolving around treatment for chronic disease states such as hypertension, diabetes, and hyperlipidemia. However, it is unclear if physicians fully grasp the extent of nonadherence within their patient populations with regard to these medications, specifically those patients enrolled in Medicare Advantage Prescription Drug (MA-PD) plans.
\end{abstract}

OBJECTIVES: To (a) determine physicians' perceptions of medication adherence among their patients enrolled in MA-PD plans and (b) compare those perceptions with actual adherence rates obtained from claims data.

METHODS: A survey was developed and administered to primary care physicians (PCPs) contracted within a Texas MA-PD plan. The previously validated questionnaire was distributed during an all-PCP quarterly meeting and was collected prior to the meeting's conclusion to increase completion and return rates. PCPs were requested to indicate what percentage of their patients they believed to be adherent to each of the CMS Part D star medication classes, which includes statins, oral antidiabetic drugs (OADs), and reninangiotensin system (RAS) antagonists; what financial category they believe the majority of their patients fall under; and what percentage of their patients receive samples. The PCPs' perceived percentage of adherent patients were compared with the calculated percentage of patients, using a chi-square test at an a priori alpha level of 0.05 . The calculated adherence was obtained from pharmacy claims data, meeting the CMS targeted adherence threshold $(\geq 80 \%$ ). This adherence rate was calculated using proportion of days covered (PDC) for all 3 medication categories in each PCP's patient population.

RESULTS: To compare PCP perception of patient adherence and actual adherence, 226 PCPs were used. The sample population shared similar sex and age distribution with the national physician average; however, there was more racial diversity represented. PCP perception of patient adherence, as well as the actual percentage of adherent patients, were significantly $(P<0.05)$ different across statins, OADs, and RAS antagonists; lowest perceived percentage, as well as actual percentage, were reported for statins. PCP perception of patient adherence and actual percentage of adherent patients were significantly different in the 3 medication categories. PCPs' correct estimations were significantly $(P<0.0001)$ lower than expected values, while over- and underestimations were significantly $(P<0.0001)$ higher than the expected values.

CONCLUSIONS: PCPs were almost equally likely to over- or underestimate percentage of adherent patients in their patient pools.

J Manag Care Spec Pharm. 2016;22(3):305-12

Copyright $\odot 2016$, Academy of Managed Care Pharmacy. All rights reserved.

\section{What is already known about this subject}

Poor prescription medication adherence leads to poor disease control, increased hospitalizations, and increased health care costs.

Medicare-eligible seniors take many prescription medications and often face many barriers that may inhibit adherence to these medications.

Physicians historically have been inaccurate at predicting patient medication adherence, with most overestimating adherence rates.

\section{What this study adds}

This study of Medicare Advantage Prescription Drug (MA-PD) plan primary care physicians provides physician perceptions of patients' medication adherence rates for statins, diabetes agents, and renin-angiotensin system antagonists.

This study compares physician's adherence estimates with actual calculated adherence rates from pharmacy claims data, providing the opportunity to determine how accurately physicians can estimate adherence rates.

This study compares estimates of Medicare beneficiary adherence with those previously published in the literature.

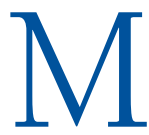
edication adherence has become a global concern in the health care industry. There is growing evidence that medication nonadherence is associated with adverse health outcomes and higher costs of care; this burden is becoming alarmingly more prevalent. ${ }^{1,2}$ One mounting area of concern is the aging population: As the population ages, more patients will likely require additional medications to treat chronic conditions. It is estimated that $90 \%$ of eligible Medicare beneficiaries are taking at least 1 prescription medication, with the average patient receiving 6-8 medications. ${ }^{3-5}$ Medication nonadherence has been associated with hospitalization rates up to $10 \%$ and contributes to about 125,000 deaths per year. ${ }^{6}$

In contrast to lower rates of adherence leading to adverse outcomes and higher costs of care, it has been documented that improvements in medication adherence have demonstrated improved health outcomes and reduced health care costs. ${ }^{7-9}$ Medication adherence has also become an important focus for the Centers for Medicare \& Medicaid Services (CMS), which 
has incorporated medication adherence into Part D quality metrics to determine volume of plan reimbursement funds. ${ }^{10}$ The Part D star measures help quantify the quality of services provided to Medicare beneficiaries, with the goal to achieve 5 stars (indicating the highest quality) in each measure. There are 3 measures related to medication adherence, with 1 to address each of the major chronic disease states (diabetes mellitus, hyperlipidemia, and hypertension). Each of the adherence measures are triple weighted, carrying a great significance in the Part D star rating program. Primary care physicians (PCPs) contracted with certain Medicare Advantage health plans may be eligible to receive quality bonus incentives for achieving such measures through predetermined thresholds established by CMS. The payer provides this award to the PCPs as achievement of the star goals that help the plan receive not only quality bonus payments from CMS, but also allows for the ability to offer more robust benefits to their plan beneficiaries. ${ }^{11}$ Thus, PCPs may have a financial interest in improving medication adherence rates among their Medicare populations.

Although most Americans agree that prescription medications are important to their health, 54\% admit that they do not consistently take their medications as prescribed. ${ }^{12}$ Studies have indicated that up to $30 \%$ of patients do not initially fill antihypertensive, lipid lowering, and oral diabetes medications, and among those that do fill a new prescription, one half discontinue the therapy within the first 6 months. ${ }^{13,14}$ Additional barriers have been identified that may hinder adequate care among the Medicare population, including financial hardships, forgetfulness, transportation issues, poor communication with providers, and incomplete understanding of diseases or drug regimen. ${ }^{15-17}$ The World Health Organization has reported that increasing the effectiveness of adherence interventions could have a far greater impact on the health of the population than any improvements in specific medical treatments. ${ }^{18}$ Many physicians do not fully grasp the extent of this problem as they continually overestimate their patients' medication adherence rates. ${ }^{19,20}$

There have been an abundance of articles published in the last 2 decades concerning medication nonadherence, but gaps persist in our ability to make demonstrable improvements on this front. ${ }^{21}$ Physicians cannot take actions to correct medication adherence if they are unaware that the problem exists. Studies assessing physician identification of nonadherence have indicated that they are no better than chance, correctly predicting adherence status less than half the time..$^{22-24}$ Because Medicare Advantage Prescription Drug (MA-PD) plans contain a much more specific population of elderly and disabled patients, their PCP interactions focus on a more limited array of disease states. It is important for MA-PD physicians to recognize nonadherence in their patient populations so that they may adequately evaluate medication regimens and ensure disease control and good health outcomes. Additionally, MA-PD physicians have a direct financial incentive to improve patient adherence due to CMS reimbursement funds. ${ }^{10,25-27}$

The objective of this study was to assess PCPs' perceptions of patient medication adherence for each of the CMS Part D star medication classes, which includes statins, oral antidiabetic drugs (OADs), and renin-angiotensin system (RAS) antagonists. We aimed to compare PCP's perceptions of the percentage of adherent patients with the actual percentage of adherent patients calculated from pharmacy claims data.

\section{Methods}

A cross-sectional survey of PCPs contracted with an MA-PD plan in Texas was conducted. A convenience sample of only PCPs within the MA-PD plan in the greater Houston, El Paso, and Rio Grande Valley regions were administered the survey. Any specialists or PCPs outside of the specified geographic regions were excluded. The paper-based survey was administered to all PCPs during a quarterly meeting. PCPs were required to attend the meeting in order to receive their annual bonus payments. With the intent to have the highest response rates in PCP groups, the survey was distributed on-site, and providers were requested to complete and return it before leaving the meeting.

An a priori sample size calculation was conducted using GPower (3.1.4; Heinrich-Heine-University, Dusseldorf, Germany), keeping the alpha level at 0.05 and power 95\%, indicated a sample size of 207 physicians to be sufficient to assess the objective of comparing perceived and actual adherence with an effect size of 0.3 .

\section{Physician Survey Development}

The survey instrument was developed by researchers upon review of existing literature. Face and content validity and reliability (test-retest method) was assessed through a focus group of 5 MA-PD plan medical directors to determine if content was adequate to obtain the necessary data. In doing so, information on redundancies and inaccuracies were identified and corrected. The same PCPs were provided the survey again to see if the scores remained the same after about a week. The study was conducted upon approval from the University of Houston Institutional Review Board. The data collection was performed internally by the MA-PD organization throughout 


\section{FIGURE 1 Survey Questions Used in This Study}

1. In your perception, which financial category best describes the majority of your [MA-PD Name] patients?

$\square$ Upper Middle Class $\square$ Middle Class $\square$ Indigent (Medicaid, low-income subsidy)

2. In your perception, what percentage (\%) of your [MA-PD Name] patients receives drug samples due to cost?

$\square<10 \% \square 10 \%-25 \% \square 26 \%-50 \% \square>50 \%$

3. CMS measures define adherence as patients taking their medication $>80 \%$ of the time. Based on this definition, please indicate the percentage (\%) of your [MA-PD Name] patients you believe to be compliant to the measure:

\begin{tabular}{lllll}
\hline a. Part D cholesterol medications (statins) & $<25 \%$ & $25 \%-49 \%$ & $50 \%-75 \%$ & $>75 \%$ \\
\hline b. Part D diabetes medications (oral diabetes agents) & $<25 \%$ & $25 \%-49 \%$ & $50 \%-75 \%$ & $>75 \%$ \\
\hline c. Part D blood pressure medications (ACE/ARBs) & $<25 \%$ & $25 \%-49 \%$ & $50 \%-75 \%$ & $>75 \%$ \\
\hline
\end{tabular}

4. Sometimes our pharmacy team will follow-up with you by phone after contacting you by fax or other means regarding Part D star measures for [MA-PD Name] patients. How receptive are you to this follow-up contact?

$\square$ Welcome/appreciate $\square$ Feel indifferent $\square$ Do not desire/feel it is not useful

$A C E=$ angiotensin-converting enzyme inhibitor; $A R B=$ angiotensin II receptor blocker; $M A-P D=$ Medicare Advantage Prescription Drug plan.

the month of December 2014; quarterly meetings took place on different days of the month and varied by geographic region. Deidentified data, along with demographic information and prescription claims information, was provided by the MA-PD organization in a combined dataset for analyses.

The survey consisted of 1 page; questions used for analysis in this study are shown in Figure 1. PCPs were asked to estimate the percentage of patients within the MA-PD plan adherent to each of the CMS star medications. The CMS definition of adherence was provided, which is taking a medication at least $80 \%$ of the time. CMS star medications include statins, OADs, and RAS antagonists. ${ }^{7}$ The scale used to measure the PCPs' perceptions of adherence had 4 options to indicate their perceived percentage of adherent patients: $<25 \%$, $25 \%-49 \%, 50 \%-75 \%$, and $>75 \%$. Further, the PCPs were given a multiple choice selection to indicate the financial category best describing the majority of their MA-PD patients, with options of upper middle class, middle class, or indigent. PCPs also provided information on the estimated percentage of their MA-PD patients who received drug samples because of high medication cost on the following scale: $<10 \%, 10 \%-25 \%, 26 \%-$ $50 \%$, and $>50 \%$. In addition, PCPs were asked how receptive they were to follow-up communication. Options were welcome follow-up communication, indifferent to the follow-up communication, or find follow-up communication not useful.

\section{Outcome Measurement}

Outcome was measured as the percentage of adherent patients, calculated as perceived and actual percentages. Perceived percentage of adherent patients was reported by the PCPs in the survey by selecting 1 of these 4 categories: $<25 \%, 25 \%$ $49 \%, 50 \%-75 \%$, and $>75 \%$. The actual percentage of adherent patients for each PCP was calculated using proportion of days covered (PDC). The PDC was calculated by dividing the total number of days supply of a medication that the patient picked up from the pharmacy (per claims data) by the total number of days in the year since the medication was first filled by the patient. ${ }^{1}$ This is the official measurement used by CMS to evaluate patient medication adherence; patients with $\mathrm{PDC} \geq 80 \%$ were defined as adherent, while patients with PDC $<80 \%$ were defined as nonadherent for the medications investigated in this study. PDC calculations were completed for each of the 3 medication classes for each of the patients covered by the responding PCPs. For each PCP patient pool, the percentage of patients achieving a $\mathrm{PDC} \geq 80 \%$ for each medication category was determined. The percentage value obtained for those adherent was then categorized into the 4 categories: <25\%, 25\%-49\%, $50 \%-75 \%$, and $>75 \%$.

PCPs were categorized into 3 groups based on the difference between their perceived and actual percentages of adherent patients: correct estimators, underestimators, and overestimators. PCPs were categorized as correct estimators if their actual amount of adherent patients from claims data fell within the range they had estimated for that medication class within the survey. For example, if a physician estimated that 50\%-75\% of patients are adherent to statins, and claims revealed that $60 \%$ of patients were adherent, then this was considered a correct estimate. PCPs with incorrect estimates were classified as underestimators if the PCP indicated a lower range of adherent patients than what actually showed from claims data, or 


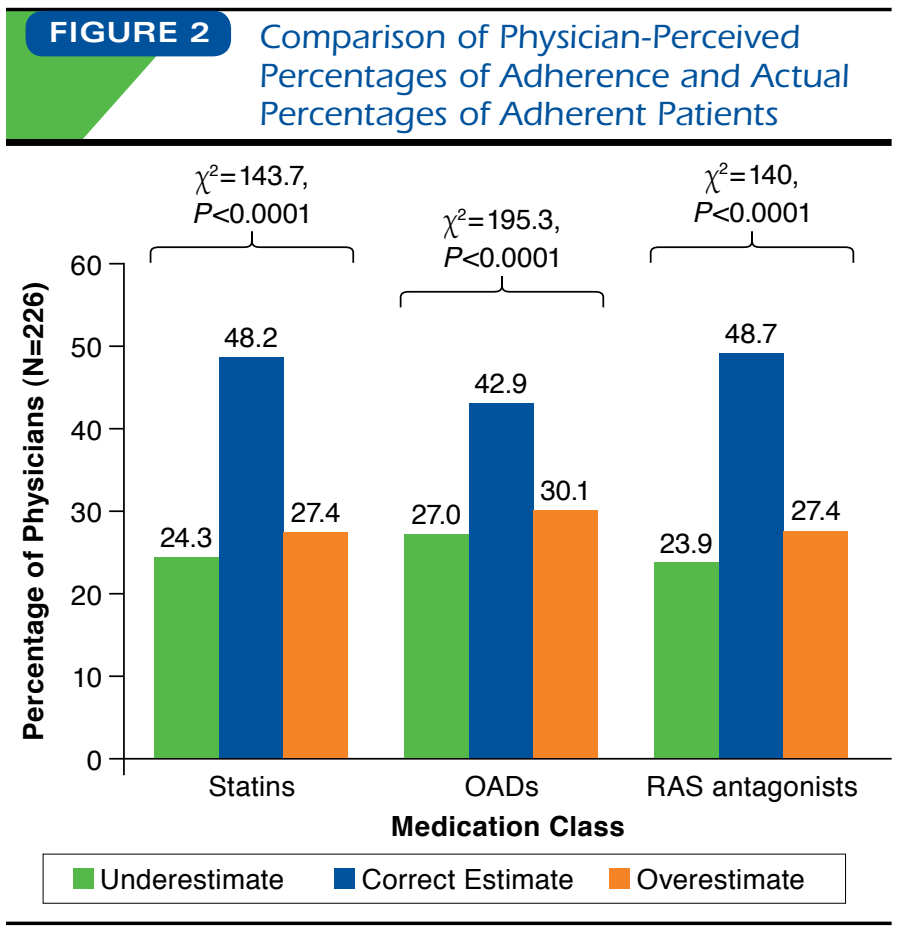

$O A D s=$ oral antidiabetic drugs; $R A S=$ renin-angiotensin system; $\chi^{2}=$ chi-square test.

overestimators if the PCP indicated a higher range of adherent patients than what actually showed from claims data. Based on anticipated norms of errors, we expected that there would be a maximum of $10 \%$ under- and overestimation variance by PCPs.

\section{Statistical Analysis}

Data were obtained and coded in a Microsoft Excel spreadsheet and were analyzed using SAS 9.3. (SAS Institute, Carey, NC). Descriptive statistics, including means and frequencies were performed to describe population characteristics and to evaluate the perceived percentage of patients' $\geq 80 \%$ PDC values to actual percentage of patients' $\geq 80 \%$ PDC values.

The accuracy of physician estimation was calculated by dividing PCPs' estimations into 3 categories. Estimation was calculated as the difference between perceived and actual patient adherence. If the perceived adherence was lower than the actual adherence, it was defined as underestimation; if the perceived adherence was higher than the actual adherence, then it was defined as overestimation. Physician estimation was compared across each MA-PD plan using a chi-square test at 0.05 alpha level (Figure 2). A chi-square test at 0.05 alpha level was used to compare the difference between perceived and actual adherence across the 3 Part D medications.

\begin{tabular}{|c|c|c|}
\hline \multirow[b]{2}{*}{ Characteristics } & \multicolumn{2}{|c|}{ Respondents, $\mathrm{N}=226$} \\
\hline & $\mathbf{n}$ & $\%$ \\
\hline \multicolumn{3}{|l|}{ Physician age group } \\
\hline $30-60$ years & 146 & 64.6 \\
\hline Greater than 60 years & 68 & 30.1 \\
\hline Unknown age & 12 & 5.3 \\
\hline \multicolumn{3}{|l|}{ Physician sex } \\
\hline Male & 152 & 67.2 \\
\hline Female & 68 & 30.1 \\
\hline Unknown & 6 & 2.7 \\
\hline \multicolumn{3}{|l|}{ Physician race } \\
\hline White & 69 & 30.5 \\
\hline Hispanic & 65 & 28.7 \\
\hline Black & 19 & 8.4 \\
\hline Asian & 41 & 18.1 \\
\hline Unknown & 32 & 14.2 \\
\hline \multicolumn{3}{|l|}{ Years in practice } \\
\hline Less than 10 years & 17 & 7.5 \\
\hline $10-25$ years & 90 & 39.8 \\
\hline Greater than 25 years & 107 & 47.3 \\
\hline Unknown & 12 & 5.3 \\
\hline \multicolumn{3}{|c|}{ Receptive to follow-up pharmacist communication } \\
\hline Welcome & 166 & 73.4 \\
\hline Indifferent & 38 & 16.8 \\
\hline Not useful & 16 & 7.0 \\
\hline No response & 6 & 2.7 \\
\hline \multicolumn{3}{|l|}{ Financial category of patients } \\
\hline Upper middle and middle class & 125 & 55.3 \\
\hline Indigent & 88 & 38.9 \\
\hline No response & 13 & 5.8 \\
\hline \multicolumn{3}{|l|}{ Medication sample use } \\
\hline$<25 \%$ of patients & 176 & 77.9 \\
\hline $26 \%-50 \%$ of patients & 38 & 16.8 \\
\hline$>50 \%$ of patients & 10 & 4.4 \\
\hline No response & 2 & 0.9 \\
\hline
\end{tabular}

\section{Results}

A total of 239 PCPs from across Texas completed and returned the survey for a response rate of $77.9 \%$. A final sample of $226(73.8 \%)$ physicians was included for analysis, since pharmacy claims data information was missing/incomplete for patients of the remaining 13 physicians. Demographic information was incomplete for some providers. Participant characteristics are summarized in Table 1. The population contained primarily males (67.2\%) and nonwhite patients (55.2\%). Age ranged from 30 to 84 years with an average age of $53(\mathrm{SD}=11.2)$ years. The number of years in practice ranged from 4 to 55 with an average of $26(S D=10.6)$ years. The age and sex of this sample were similar to the national average, although there was more racial diversity represented in our study, with 


\section{TABLE 2 Comparison of Physician-Perceived and Actual Percentage of Adherent Patients Across} Part D Medications

\begin{tabular}{|c|c|c|c|c|c|c|c|c|c|c|}
\hline \multirow{3}{*}{$\frac{\text { Part D Medications }}{\text { Statins }}$} & \multicolumn{5}{|c|}{$\begin{array}{l}\text { Physician-Perceived Percentage } \\
\text { of Adherent Patients, } n(\%)^{a} \\
n=226\end{array}$} & \multicolumn{4}{|c|}{$\begin{array}{l}\text { Actual Percentage } \\
\text { of Adherent Patients by Physician, } n(\%)^{b} \\
n=226\end{array}$} & \multirow{2}{*}{$\begin{array}{l}\text { Average PDC } \\
\text { Values } \\
\mathbf{n}=226\end{array}$} \\
\hline & \multicolumn{2}{|c|}{$<50 \%$ c } & \multicolumn{2}{|c|}{$50 \%-75 \%$} & $>75 \%$ & \multicolumn{2}{|c|}{$<50 \%$} & $50 \%-75 \%$ c & $>75 \%$ & \\
\hline & 19 & $(8.4)$ & 119 & $(52.6)$ & $\begin{array}{ll}88 & (38.9) \\
\end{array}$ & & $(4.8)$ & $134 \quad(59.2)$ & $81 \quad(35.8)$ & $84.0 \quad( \pm 4.7)$ \\
\hline OADs & 17 & $(7.5)$ & & $(12.1)$ & $(56.1)$ & 7 & (3.0) & $100 \quad(44.2)$ & $(52.6)$ & $( \pm 5.3)$ \\
\hline RAS antagonists & 12 & (5.3) & 82 & $(36.2)$ & $(58.4)$ & 3 & $0.01)$ & $99 \quad(43.8)$ & $124 \quad(54.8)$ & $( \pm 3.8)$ \\
\hline
\end{tabular}

Note: There was no statistically significant difference between the perceived and actual percentage of adherent patients within each Part $D$ medication.

${ }^{a}$ Reported by physicians in the survey; perceived percentage of adherent patients varied significantly (chi-square test $\left.=21.33, P<0.05\right)$ between the Part $D$ medications.

${ }^{b}$ Calculated from pharmacy claims of patients with each of the physicians; actual percentage of adherent patients varied significantly (chi-square test $\left.=21.96, P<0.05\right)$

between the Part D medications.

${ }^{c}$ Categories $<25 \%$ and $25 \%-49 \%$ were combined because of low percentage of adherent patients in those categories.

${ }^{d}$ Average PDC values were calculated using the sum of PDC values for the patients divided by the number of patients (PDC calculated from pharmacy claims data).

OADs = oral antidiabetic drugs; PDC= proportion of days covered; RAS = renin-angiotensin system.

more black, Asian, and Hispanic physicians and less white physicians than the national average..$^{28,29}$

Physician responses concerning sample use and categorization of patient financial situation are summarized in Table 1. Most PCPs estimated that the majority of their MA-PD patients were upper middle class/middle class (55.3\%) versus indigent (38.9\%). About $75 \%$ of physicians welcomed follow-up communication from the health plan. Most PCPs reported that $<25 \%$ of their patients receive medication samples (77.9\%).

\section{Actual and Perceived Adherence}

Table 2 depicts a comparison of physician-perceived adherence and actual adherence across the 3 medication classes. For reporting percentages of perceived and actual adherent patients, the categories $<25 \%$ and $25 \%-49 \%$ were combined into a single category $(<50 \%)$ because of low sample size in each. Thus, the results report percentage of adherent patients across 3 categories: $<50 \%, 50 \%-75 \%$, and $>75 \%$ across the 3 medication categories. Perceived proportion of adherent patients differed significantly across statins, OADs, and RAS antagonists $\left(\chi^{2}=21.33, P<0.05\right)$. Among the medication classes compared, PCPs perceived that statin users had the lowest proportion of patients with $>75 \%$ adherence (38.9\%), compared with OADs (56.1\%) and RAS antagonists (58.4\%). Actual proportion of adherent patients also differed significantly across statins, OADs, and RAS antagonists $\left(\chi^{2}=21.96, P<0.05\right)$, with the lowest percentage of adherent patients among statin users.

When the PCP-perceived percentages of adherent patients were categorized as underestimates, correct estimates, and overestimates and compared with the actual percentage for each of the 3 medication categories, nearly half of the PCPs did not correctly estimate $(P<0.0001)$ the proportion of adherent patients: statins 48.2\%, OADs $42.9 \%$, and RAS antagonists $48.7 \%$ (Figure 2). Among PCPs that incorrectly estimated adherence rates, a higher proportion overestimated $(27.4 \%, 30.1 \%, 27.4 \%$ for statins, OADs, and RAS antagonists, respectively) than underestimated $(24.3 \%, 27 \%, 23.9 \%$ for statins, OADs, and RAS antagonists, respectively) at $P=0.05$.

\section{Discussion}

Our study provides valuable information regarding PCPs' perceptions of adherence in MA-PD patients. The PCPs surveyed were highly motivated to improve adherence scores within their MA-PD populations and provided extraordinarily high response rates. Average published physician response rates to survey questionnaires hover in the 50\% range, with the general consensus being that surveys with a lower than 50\% response rate are unlikely to reach publication. ${ }^{30,31}$ Literature suggests that providing tangible incentives or rewards for survey participation can help increase response rates. ${ }^{32} \mathrm{~A}$ response rate of $78 \%$ was achieved without the use of direct incentives. A statement was included in the survey instructions indicating the goal of improving patient medication adherence. Part D star ratings and potential for quality bonus incentives appear to be motivation enough for MA-PD PCPs to provide feedback via survey response.

Use of medication samples is an important item to quantify because these samples are not captured in pharmacy claims and so are not reflected in PDC calculations. Use of samples may detract from PCP adherence rates and CMS star ratings, although they may be necessary to allow for continuation of therapy in patients who reach the coverage gap or cannot afford 
a particular medication. Previous studies of Medicare beneficiaries have reported medication sample use as high as 38\%. ${ }^{33}$ In a 2002 survey of Medicare beneficiaries, roughly 30\% of respondents reported acquiring samples from their physicians to offset costs; a greater percentage of patients who fell into the coverage gap reported sample use over those who did not reach the gap that year. ${ }^{34}$ In our study, the majority of responding PCPs indicated that $<25 \%$ of their patients use samples. It is not surprising that since the advent of the PCP star incentives in 2011, reported sample use has declined within the MA-PD population. While adherence is a heavily weighted CMS metric, it is important to remember that blood pressure and blood sugar control are also evaluated by CMS and play a part in the reimbursement incentive, so PCPs are motivated to provide samples as necessary for disease control.

Of the 3 Part D star medication classes, physicians indicated that statins may be the most difficult class in achieving adherence. The majority of physicians believed that $>75 \%$ of their patients are adherent to RAS antagonists and diabetes agents, but that only $50 \%-75 \%$ are adherent to statins. A 2006 Internet-based survey of physicians revealed $88 \%$ of respondents believed that patients with chronic illnesses are adherent to medication about half the time or less. ${ }^{35}$ However, the same study included practitioners across all specialties, treating patients of all ages, in a hospital setting and thus may not directly translate to our study population. Patients have reported various reasons for not taking statin medications, with the most common responses being adverse effects, not feeling a benefit from the drug, concern about adverse effects, and preferring to try lifestyle modifications. ${ }^{36}$ The thresholds used for the 2014 MA-PD star ratings validate the increased difficulty in statin adherence over the other medication classes, since the 5-star cutoff for RAS antagonists was $79 \%$, diabetes $77 \%$, and statins $75 \% .^{37}$

The PCPs in this study population did not differ from previously published studies, showing that physicians correctly estimate adherence about half the time. ${ }^{22}$ Similarly, the physicians are more prone to overestimating patient adherence than underestimating. ${ }^{19,20}$ These PCPs may be frequently reminded of the burden of medication nonadherence among their patient populations through Part D presentations, targeted one-onone interactions with others from the MA-PD plan, as well as receiving educational materials and performance reporting tools. These frequent reinforcements may contribute to a decreased confidence in patient adherence, leading to a decrease in overestimation of adherence rates.

Osterberg and Blaschke (2005) noted that physicians' ability to recognize nonadherence is generally poor. ${ }^{1}$ Our survey actually contests this notion to a point. Yet, it is important to note that physician engagement, through education and counseling with patients, is critical to improving adherence rates, and strong physician-patient relationships where communication is heightened plays a role in the patient's view of the medication's importance and value. ${ }^{1}$ Through this survey, physicians (and pharmacists assisting with the care of a specific patient population) can evaluate PCP performance by assessing their patient population's adherence rates and target certain patients to provide needed education that may be lacking or was not perceived to have been necessary at the time of the office visit. Evaluating perceived versus actual adherence rates can help PCPs tailor the educational portion of their discussions with patients to ultimately impact outcomes.

\section{Limitations}

There are some limitations to this study. The sample size may not have been large enough to demonstrate existing relationships between physician demographics and estimates of adherence. An a priori sample size calculation was done to study the difference in physician-perceived and actual adherence. Additionally, the use of a single MA-PD plan within the state of Texas may limit the generalizability of results. Pharmacy claims data were used to determine the actual adherence rates, although claims data are not $100 \%$ accurate, since patients may fill medications outside of their insurance plan; incorrect days supply information may cause fills to last longer or shorter than expected; and medication sample use is not captured or taken into account. Finally, the questionnaire did not include a specified time frame for adherence estimates. CMS calculates adherence on a calendar year basis for star rating purposes. This was the intended time frame; however, this was not specified to the physicians completing the survey.

\section{Conclusions}

The results of this study demonstrate that PCPs within a MA-PD plan are highly motivated to provide survey feedback in order to impact patient medication adherence. When these PCPs do not correctly estimate medication adherence, they may be more likely to overestimate adherence rates. This suggests that this population of physicians is confident in patients' abilities to take medications as prescribed. MA-PD PCPs believe that patients are more adherent to RAS antagonists and diabetes agents than to statin medications. Patient education efforts may be an important step in overcoming barriers to statin adherence. Pharmacists are well equipped to assist in feedback regarding medication adherence rates, implementing initiatives for adherence improvement, and educating patients on the necessity for medication, all of which may have an impact on the Part D star ratings as well as patient outcomes. 


\section{Authors}

AMANDA WINTERS, PharmD, is Clinical Pharmacist, Pharmacy Department; TARA ESSE, PharmD, BCACP, is Clinical Program Manager, Pharmacy Department; and OMAR SERNA, PharmD, $B C A C P$, is Clinical Program Manager, Cigna-HealthSpring,

Houston, Texas. ARCHITA BHANSALI, MS, is a Graduate Student, Clinical Sciences and Administration; SHIVANI MHATRE, PhD, is a Graduate Student; and SUJIT SANSGIRY, PhD, is Associate Professor, University of Houston College of Pharmacy, Houston, Texas.

AUTHOR CORRESPONDENCE: Amanda Winters, PharmD, Pharmacy Department, Cigna-HealthSpring, 13325 Hargrave Rd., Ste. 180, Houston, TX 77070. Tel.: 281.414.8337;

E-mail: amanda.winters@healthspring.com.
10. Academy of Managed Care Pharmacy, American Pharmacists Association. Medicare star ratings: stakeholder proceedings on community pharmacy and managed care partnerships in quality. J Am Pharm Assoc (2003). 2014:54(3):228-40.

11. Centers for Medicare \& Medicaid. News flash: summary information regarding Medicare's primary care incentive payment program (PCIP). Medical Learning Network. SE No. 1109. January 1, 2011. Updated April 30, 2014. Available at: http://www.cms.gov/Outreach-and-Education/MedicareLearning-Network-MLN/MLNMattersArticles/downloads/se1109.pdf. Accessed December 21, 2015.

12. Prescription Solutions and the National Council on Patient Information and Education (NCPIE). New survey: more than half of Americans do not take prescription medicines as instructed, pointing to growing public health problem. November 12, 2009. Available at: http://www.talkaboutrx.org/documents/AdherenceSurveyMediaRelease.pdf. Accessed December 20, 2015.

13. Fischer MA, Stedman MR, Lii J, et al. Primary medication nonadherence: analysis of 195,930 electronic prescriptions. J Gen Intern Med. 2010;25(4):284-90.

14. McHorney CA. The Adherence Estimator: a brief, proximal screener for patient propensity to adhere to prescription medications for chronic disease. Curr Med Res Opin. 2009;25(1):215-38.

15. Nam S, Chesla C, Stotts NA, Kroon L, Janson SL. Barriers to diabetes management: patient and provider factors. Diabetes Res Clin Pract. 2011;93(1):1-9.

16. Jerant AF, von Friederichs-Fitzwater MM, Moore M. Patients' perceived barriers to active self-management of chronic conditions. Patient Educ Couns. 2005;57(3):300-07.

17. Doucette WR, Nevins J, McDonough RP. Factors affecting collaborative care between pharmacists and physicians. Res Social Adm Pharm. 2005;1(4):565-78

18. Burkhart PV, Sabaté E. Adherence to long-term therapies: evidence for action. J Nurs Scholarsh. 2003;35(3):207.

19. Copher R, Buzinec P, Zarotsky V, Kazis L, Iqbal SU, Macarios D. Physician perception of patient adherence compared to patient adherence of osteoporosis medications from pharmacy claims. Curr Med Res Opin. 2010;26(4):777-85.

20. Trindade AJ, Ehrlich A, Kornbluth A, et al. Are your patients taking their medicine? Validation of a new adherence scale in patients with inflammatory bowel disease and comparison with physician perception of adherence. Inflamm Bowel Dis. 2011;17(2):599-604.

21. Seabury SA, Gupta CN, Philipson TJ, Henkhaus LE. Understanding and overcoming barriers to medication adherence: a review of research priorities J Manag Care Spec Pharm. 2014;20(8):775-83. Available at: http://www.jmcp. org/doi/abs/10.18553/jmcp.2014.20.8.775

22. Meddings J, Kerr EA, Heisler M, Hofer TP. Physician assessments of medication adherence and decisions to intensify medications for patients with uncontrolled blood pressure: still no better than a coin toss. BMC Health Serv Res. 2012;12:270.

23. Gilbert JR, Evans CE, Haynes RB, Tugwell P. Predicting compliance with a regimen of digoxin therapy in family practice. Can Med Assoc J. 1980;123(2):119-22.

24. Mushlin AI, Appel FA. Diagnosing potential noncompliance. Physicians' ability in a behavioral dimension of medical care. Arch Intern Med. 1977;137(3):318-21.

25. Bramley TJ, Gerbino PP, Nightengale BS, Frech-Tamas F. Relationship of blood pressure control to adherence with antihypertensive monotherapy in 13 managed care organizations. J Manag Care Pharm. 2006;12(3):239-45. Available at: http://amcp.org/data/jmcp/Research_239-245.pdf.

26. Ho PM, Rumsfeld JS, Masoudi FA, et al. Effect of medication nonadherence on hospitalization and mortality among patients with diabetes mellitus. Arch Intern Med. 2006;166(17):1836-41. 
27. Rasmussen JN, Chong A, Alter DA. Relationship between adherence to evidence-based pharmacotherapy and long-term mortality after acute myocardial infarction. JAMA. 2007;297(2):177-86.

28. Young A, Chaudhry HJ, Pei X, et al. A census of actively licensed physicians in the United States, 2014. J Med Reg. 2015;101:8-23.

29. Association of American Medical Colleges (AAMC). Diversity in the physician workforce: facts and figures 2014. Available at: http://aamcdiversityfactsandfigures.org/section-v-additional-detailed-tables/. Accessed December $20,2015$.

30. Kellerman SE, Herold J. Physician response to surveys. A review of the literature. Am J Prev Med. 2001;20(1):61-67.

31. James KM, Ziegenfuss JY, Tilburt JC, Harris AM, Beebe TJ. Getting physicians to respond: the impact of incentive type and timing on physician survey response rates. Health Serv Res. 2011;46(1 Pt 1):232-42.

32. Vangeest JB, Johnson TP, Welch VL. Methodologies for improving response rates in surveys of physicians: a systematic review. Eval Health Prof. 2007;30(4):303-21.
33. Cox ER, Jernigan C, Coons SJ, Draugalis JL. Medicare beneficiaries' management of capped prescription benefits. Med Care. 2001;39(3):296-301.

34. Tseng CW, Brook RH, Keeler E, Steers WN, Mangione CM.

Cost-lowering strategies used by medicare beneficiaries who exceed drug benefit caps and have a gap in drug coverage. JAMA. 2004;292(8):952-60.

35. Patel UD, Davis MM. Physicians' attitudes and practices regarding adherence to medical regimens by patients with chronic illness. Clin Pediatr (Phila). 2006;45(5):439-45.

36. Harrison TN, Derose SF, Cheetham TC, et al. Primary nonadherence to statin therapy: patients' perceptions. Am J Manag Care. 2013; 19(4):e133-e39.

37. Centers for Medicare $\&$ Medicaid Services. Part $C$ and D performance data. 2014 Part C \& D Medicare star ratings data. Available at: http://www. cms.gov/Medicare/Prescription-Drug-Coverage/PrescriptionDrugCovGenIn/ PerformanceData.html. Accessed December 20, 2015. 